The role of helium implantation induced vacancy defect on hardening of tungsten

Xin Ou', Wolfgang Anwand', Reinhard Kögler', Hong-Bo Zhou, and Asta Richter'

Citation: Journal of Applied Physics 115, 123521 (2014); doi: 10.1063/1.4870234

View online: http://dx.doi.org/10.1063/1.4870234

View Table of Contents: http://aip.scitation.org/toc/jap/115/12

Published by the American Institute of Physics

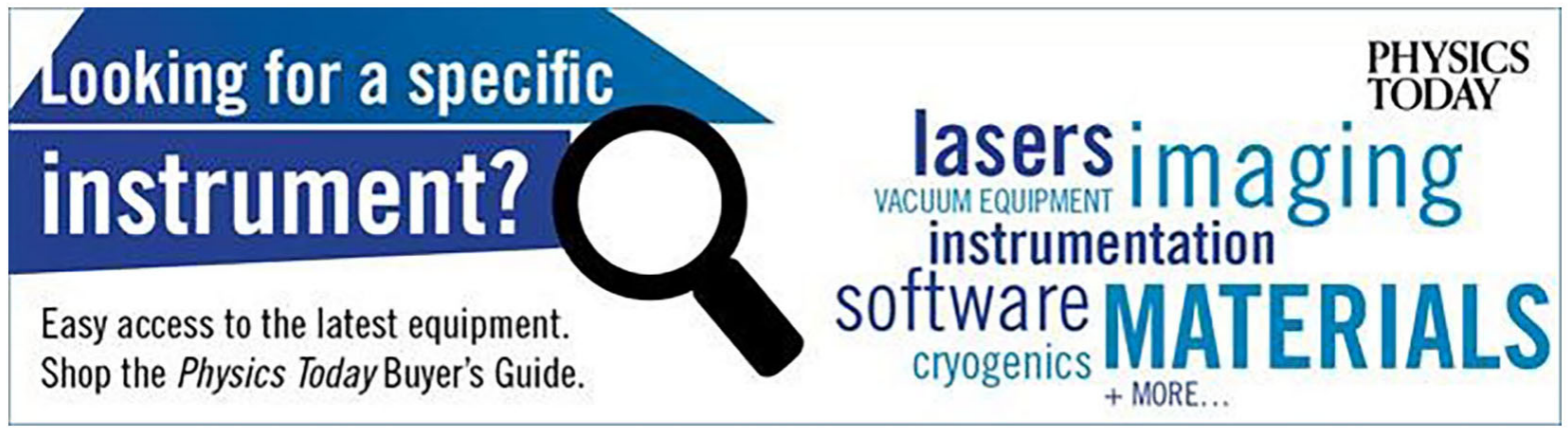




\title{
The role of helium implantation induced vacancy defect on hardening of tungsten
}

\author{
Xin Ou, ${ }^{1,2, a)}$ Wolfgang Anwand, ${ }^{1, b)}$ Reinhard Kögler, ${ }^{1, c)}$ Hong-Bo Zhou, ${ }^{3}$ \\ and Asta Richter ${ }^{4, d)}$ \\ ${ }^{1}$ Helmholtz-Zentrum Dresden-Rossendorf, Bautzner Landstr. 400, 01328 Dresden, Germany \\ ${ }^{2}$ State Key Laboratory of Functional Material for Informatics, Shanghai Institute of Microsystem and \\ Information Technology, Chinese Academy of Sciences, Shanghai 200250, China \\ ${ }^{3}$ Department of Physics, Beihang University, Beijing 100191, China \\ ${ }^{4}$ Technische Hochschule Wildau, Hochschulring1, 15745 Wildau, Germany
}

(Received 12 February 2014; accepted 21 March 2014; published online 28 March 2014)

\begin{abstract}
Vacancy-type defects created by helium implantation in tungsten and their impact on the nano-hardness characteristics were investigated by correlating the results from the positron annihilation spectroscopy and the nano-indentation technique. Helium implantation was performed at room temperature (RT) and at an elevated temperate of $600{ }^{\circ} \mathrm{C}$. Also, the effect of post-annealing of the RT implanted sample was studied. The S parameter characterizing the open volume in the material was found to increase after helium irradiation and is significantly enhanced for the samples thermally treated at $600{ }^{\circ} \mathrm{C}$ either by irradiation at high temperature or by post-annealing. Two types of helium-vacancy defects were detected after helium irradiation; small defects with high helium-to-vacancy ratio (low S parameter) for RT irradiation and large defects with low helium-to-vacancy ratio (high $\mathrm{S}$ parameter) for thermally treated tungsten. The hardness of the heat treated tungsten coincides with the $\mathrm{S}$ parameter, and hence is controlled by the large helium-vacancy defects. The hardness of tungsten irradiated at RT without thermal treatment is dominated by manufacturing related defects such as dislocation loops and impurity clusters and additionally by trapped He atoms from irradiation effects, which enhance hardness. He-stabilized dislocation loops mainly cause the very high hardness values in RT irradiated samples without post-annealing. @ 2014 AIP Publishing LLC. [http://dx.doi.org/10.1063/1.4870234]
\end{abstract}

\section{INTRODUCTION}

There is a significant interest in nuclear fusion energy for electricity generation, because it is a clean, safe, and environmental friendly energy source. Structural materials for the fusion energy system require excellent performance in high heat flux deposition and particles irradiation. Tungsten as a high- $\mathrm{Z}$ material is a promising candidate for the plasma-facing materials. Tungsten has a low sputtering yield for the light elements, a high thermal conductivity, high mechanical strength, and a high melting temperature $(3680 \mathrm{~K})$. Due to these intrinsic physical properties, tungsten has been selected as the plasma-facing material in the experimental fusion reactor ITER and DEMO. ${ }^{1}$ In the nuclear reactors, the materials structure suffers from the intensive irradiation environment. The major irradiation effect comes from the neutron and $\alpha$ particle irradiation. A simulation of the irradiation damage induced by energetic particles in the reactors can be achieved by applying the energetic ion beam irradiation (IBI). IBI is a feasible and efficient tool to investigate the radiation damage in nuclear materials. ${ }^{2-5}$

Helium ion implantation was used to simulate $\alpha$ particle irradiation effects on materials considered for applications in fusion reactors. ${ }^{6-8}$ Because the surface erosion of tungsten is

\footnotetext{
a)x.ou@hzdr.de

b)w.anwand@hzdr.de

c)r.koegler@hzdr.de

d)asta.richter@th-wildau.de
}

the major concern in the fusion reactors, high flux, high fluence, and low energy (hundred eV) helium implantation was performed into tungsten at temperatures above $400^{\circ} \mathrm{C} .{ }^{9,10}$ Recently, in order to investigate the early stage of helium irradiation effect on tungsten, Lhuillier et al. ${ }^{7}$ performed irradiation with $\mathrm{He}$ ions of relatively low flux, low fluence and analyzed the corresponding microstructure of tungsten. The existence of helium in tungsten results in the formation of open volume defects such as vacancies, vacancy clusters, voids, and bubbles. At room temperature (RT), the migration energy of $\mathrm{He}$ interstitials in tungsten is as low as $0.3 \mathrm{eV}$. $^{11}$ $\mathrm{He}$ atoms are highly mobile in defect-free tungsten and they tend to aggregate and to be trapped by the open volume defects in the tungsten matrix. Therefore, He-vacancy complexes are created in the form of $\mathrm{He}_{\mathrm{n}} \mathrm{V}_{\mathrm{m}}$. Moreover, there is a "self-trapping" effect for the He atoms in tungsten. Due to their large binding energy of the order of $1 \mathrm{eV}$, the implanted $\mathrm{He}$ can form interstitial He-He clusters in tungsten. This effect results in the surface accumulation of $\mathrm{He}$ in implanted tungsten. ${ }^{12,13}$ When the number of He atoms in the clusters is increased, the surrounding tungsten atoms can be displaced from their lattice sites and $\mathrm{He}_{\mathrm{n}} \mathrm{V}_{\mathrm{m}}$ defects are created due to the enhanced pressurization. ${ }^{14}$ The open volume defects, especially the helium-filled bubbles $\left(\mathrm{He}_{\mathrm{n}} \mathrm{V}_{\mathrm{m}}\right)$, play a significant role in deteriorating of the mechanical properties of tungsten and result in the surface swelling and embrittlement of the material. ${ }^{14}$ Therefore, the investigation of the open volume defect formation in helium implanted tungsten 
and its role on the hardening characteristics will contribute to a better understanding of the thermo-mechanical behavior of tungsten under helium irradiation. Most of the peer reports are focused on the study of the evolution of the defects in the structural materials induced by helium implantation, for instance the helium-vacancy defects, ${ }^{6-8}$ and very few works ${ }^{15}$ have demonstrated the modification of macroscopic materials properties, especially the mechanical properties, resulting from such defect formation. As the helium bubbles grow at certain conditions, severe blistering of the surface layer may occur. ${ }^{16}$ This is another critical irradiation problem in addition to the surface erosion of tungsten, and intensive investigations are desired. In this work, the irradiation damage induced by helium implantation was investigated by a correlation between the microstructure analyses of vacancy defects via positron annihilation spectroscopy (PAS) and the hardness measurements via nano-indentation (NI).

\section{EXPERIMENT DETAILS}

\section{A. Sample preparation}

Pieces of $150 \mu \mathrm{m}$ thick polycrystalline tungsten foils with a purity of $99.9 \%$ were used for this study. Helium implantations were performed at a fixed incidence angle of $22.5^{\circ}$ by $500 \mathrm{kV}$ implanter in dual ion beam facility (angle of two beam lines is $45^{\circ}$ ) of the Helmholtz-Zentrum DresdenRossendorf (HZDR). Low fluence helium implantation into tungsten with the ion energy of $350 \mathrm{keV}$ was performed at room temperature and at elevated temperature of $600^{\circ} \mathrm{C}$. For this ion energy, the bubbles are formed in tungsten in the whole range from the surface up to few hundred $\mathrm{nm}$. The helium fluences amount to $2 \times 10^{15} \mathrm{~cm}^{-2}, 5 \times 10^{15} \mathrm{~cm}^{-2}$, and $1 \times 10^{16} \mathrm{~cm}^{-2}$, respectively. The helium ion flux is around $2 \times 10^{12} \mathrm{~cm}^{-2} \mathrm{~s}^{-1}$. A piece of each room-temperature implanted sample was post-annealed (PA) at $600^{\circ} \mathrm{C}$ for $1 \mathrm{~h}$ in vacuum. This annealing cycle was chosen to be comparable with the thermal processing cycle of high temperature implantation.

\section{B. Positron annihilation spectroscopy}

The formation of open volume defects induced by $\mathrm{He}$ implantation, their growth at temperatures of $600^{\circ} \mathrm{C}$, and the capture of $\mathrm{He}$ atoms in these defects were investigated by positron annihilation Doppler broadening spectroscopy (DBS). DBS measurements were carried out with the monoenergetic positron beam "SPONSOR" at HZDR ${ }^{17}$ at which a variation of the positron energy E from $30 \mathrm{eV}$ to $36 \mathrm{keV}$ with a smallest step width of $50 \mathrm{eV}$, if required, is possible. The energy resolution of the $\mathrm{Ge}$ detector at $511 \mathrm{keV}$ is $(1.09+0.01) \mathrm{keV}$, resulting in a high sensitivity to changes in material properties from surface to depth. About $3 \times 10^{5}$ events per spectrum were accumulated. DBS is an excellent technique to detect clusters consisting of several vacancies down to open volume defects smaller than a mono-vacancy. The positron in a crystal lattice is strongly subjected to a repulsion from the positive atom core. Because of the locally reduced atomic density inside the open volume defects, with a lower local electron density, positrons have a high probability to be trapped and to annihilate with electrons in these defects by the emission of two $511 \mathrm{keV}$ photons. Monitoring of the $511 \mathrm{keV}$ annihilation-radiation was performed by DBS. The Doppler broadening of the $511 \mathrm{keV}$ annihilation line is mainly caused by the momentum of the electron due to the very low momentum of the thermalized positron. There are two main parameters, $\mathrm{S}$ (shape) and $\mathrm{W}$ (wing), obtained from the DBS. The S parameter is defined as the ratio of the counts from the central part of the annihilation peak (here $510.03-511.97 \mathrm{keV}$ ) to the total number of counts in the whole peak $(498.5-523.5 \mathrm{keV})$. The $\mathrm{W}$ parameter is defined as the ratio of counts in the two wings of the annihilation spectrum (513.16-514.13 keV and 507.87$508.84 \mathrm{keV}$ ) to the total number of counts in the peak. The $\mathrm{S}$ parameter reflects the fraction of positrons annihilation with electrons of low momentum (valance electrons), and the $\mathrm{W}$ parameter corresponds with the annihilation with the high momentum electrons (core electrons). The $\mathrm{S}$ parameter is a measure for the open volume in the material, whereas the $\mathrm{W}$ parameter describes the surrounding area of the open volume.

\section{Nano-indentation}

The hardness of tungsten samples was investigated by NI. ${ }^{18,19}$ NI is a suitable method to determine the mechanical properties of a thin irradiated surface layer, since depthdependent mechanical quantities such as hardness and indentation modulus are obtained. NI measurements were performed at room temperature using the electrostatic transducer of the UBI Hysitron triboscope with a $90^{\circ}$ cube corner tip. Hardness measurements of a thin layer of hard material on top of a softer matrix material represent a complex layer system, which is very sensitive for the softer matrix unless the indenter penetrates the overlying hard layer. To avoid this effect, the indentation depth should be less than $10 \%$ of the hard layer thickness (Bückle rule). It means an indentation depth less than $60 \mathrm{~nm}$ according to the ion range profile simulated with the computer code of transport of ions in matter TRIM. ${ }^{20}$ Hardness values at very shallow penetration depth cannot be obtained with high accuracy; therefore, measurements have been performed up to a depth of $120 \mathrm{~nm}$ with an applied maximum load of $3 \mathrm{mN}$ or less. Thus, the hardness depth profile is smeared out and the indentation depth scale does not necessarily represent the real depth profile. These problems to be considered by measuring the hardness of a narrow implanted layer are well addressed in Ref. 21.

Depth dependent mechanical properties were obtained by multi-cycling indents with repeated loading and unloading at the same location on the sample surface. ${ }^{22}$ The set of data includes the entire material response, from the first indenter-to-sample contact down to the maximum penetration depth. All load-displacement curves were analyzed according to the standard Oliver-Pharr method. ${ }^{19}$ The NI measurements were performed before and after ion implantation. It was important also to measure the reference hardness values of the virgin and the non-irradiated annealed tungsten in order to determine the relevant effect of He irradiation. 


\section{RESULTS AND DISCUSSION}

\section{A. TRIM calculation}

Tungsten lattice atoms are displaced by the He implantation with ion energy of $350 \mathrm{keV}$ and Frenkel pairs, vacancies and interstitials, are created. A total number of 130 vacancies per implanted $\mathrm{He}$ ion is calculated by TRIM 98 . The total amount of implantation-induced vacancies increases with increasing He fluence. A TRIM simulation does not consider the migration of defects and the recombination of vacancies with interstitials. Therefore, this result only indicates the maximum of vacancies which are present in irradiated tungsten. Figure 1 shows calculated depth profiles of the implantation-induced vacancies and of the implanted $\mathrm{He}$ atoms. Both profiles are slightly shifted against each other. In line with the paper of Cui et al., ${ }^{15}$ the local ratio of $\mathrm{He}$ atoms and implantation-generated vacancies is given. This ratio is important for the formation of heliumvacancy $\left(\mathrm{He}_{\mathrm{n}} \mathrm{V}_{\mathrm{m}}\right)$ clusters. ${ }^{7,8,15}$ The ratio reveals that there are more vacancies per $\mathrm{He}$ atom in the region closer to the surface. Thus, He is trapped there by vacancies with higher probability. At the end of the ion range, the number of available vacancies is very low. Hence, at this depth position, the self-trapping of $\mathrm{He}$ atoms and the formation of He interstitial cluster are more probable. He diffusion deeper into the bulk is rather unlikely.

\section{B. PAS results}

The measured $\mathrm{S}$ parameters as a function of the positron energy, $S(E)$, are presented in Fig. 2 for virgin tungsten and for tungsten irradiated with $\mathrm{He}$ ions at different fluences. Irradiation was performed at both RT and elevated temperature of $600^{\circ} \mathrm{C}(\mathrm{HT})$. The $\mathrm{S}(\mathrm{E})$ plot of the un-irradiated virgin sample in Fig. 2(a) shows a small peak around the positron energy of $10 \mathrm{keV}$. This peak is attributed to pre-existing vacancy-type defects in the surface region of the tungsten sample mainly dislocation loops and impurity clusters which were generated by the sample manufacturing and surface

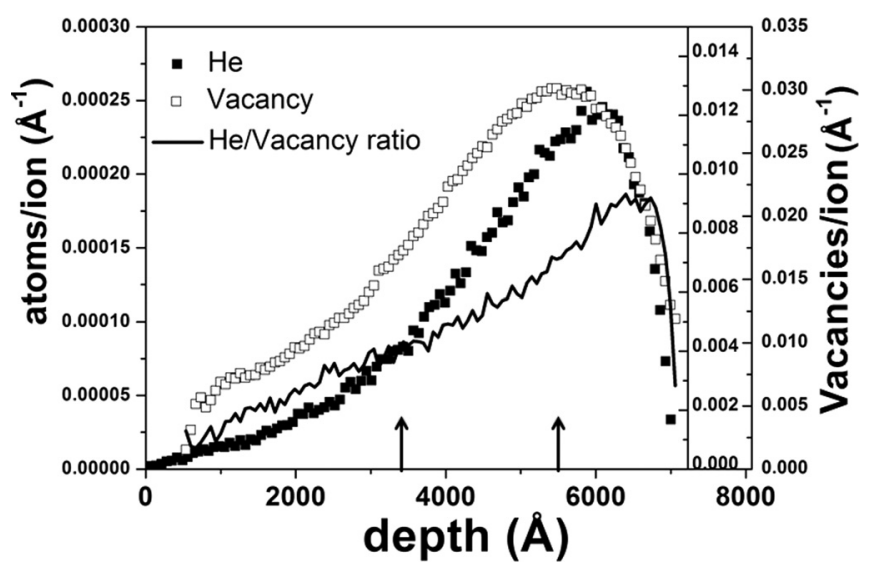

FIG. 1. TRIM simulation of depth profiles of He atoms (left scale) and tungsten vacancies (right second scale) depth profiles for $350 \mathrm{keV}$ He implantation into tungsten under incidence angle of $22.5^{\circ}$. Moreover, the local ratio of the two profiles (He/vacancy, right first scale) is shown. Arrows at the depth positions of $240 \mathrm{~nm}$ and $540 \mathrm{~nm}$ indicate the extensions of the low He-to-vacancy area and the high He-to-vacancy area, respectively.
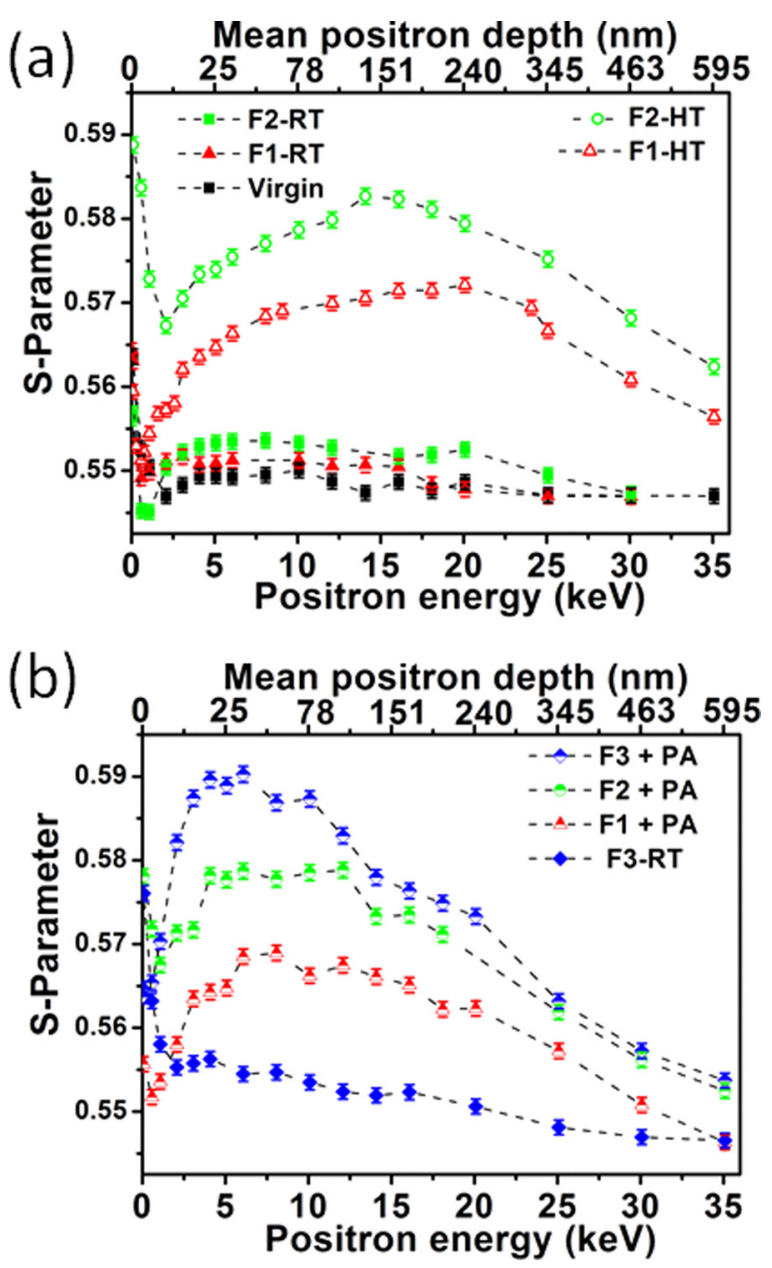

FIG. 2. S parameters as a function of the incident positron energy for virgin tungsten and $\mathrm{He}$ irradiated tungsten at RT and at $600^{\circ} \mathrm{C}(\mathrm{HT})$ (a); for tungsten $\mathrm{He}$ irradiated and post-annealed at $600^{\circ} \mathrm{C}$ (PA) (b). The irradiation fluences are $2 \times 10^{15} \mathrm{~cm}^{-2}(\mathrm{~F} 1), 5 \times 10^{15} \mathrm{~cm}^{-2}(\mathrm{~F} 2)$, and $1 \times 10^{16} \mathrm{~cm}^{-2}(\mathrm{~F} 3)$. Post-annealing of He irradiated sample at RT is performed under vacuum at $600{ }^{\circ} \mathrm{C}$ for $1 \mathrm{~h}$. The mean positron penetration depth in tungsten is shown on the top scale.

polishing. The thickness of this damaged surface layer could be determined to be $(140+41) \mathrm{nm}$. For higher positron energies, the S parameter converges to the bulk value of 0.547 .

After performing the He irradiation at room temperature, the $\mathrm{S}$ parameter peaks around $10 \mathrm{keV}$ become slightly higher and broader and the peaks increase with increasing He fluence. Broader peaks indicate a deeper reaching defect distribution. An increasing S parameter corresponds with larger size and higher concentration of the defects. However, the increase of the $\mathrm{S}$ parameter is less than expected for vacancies in tungsten ${ }^{7}$ which were created by the He implantation. This fact can be explained by a He content inside the vacancies which prevents the annihilation with the low momentum electrons of tungsten and promotes the positron annihilation with $\mathrm{He}$ electrons having higher momenta. As a consequence of this, the Doppler broadening of the annihilation line increases and the $\mathrm{S}$ parameter decreases, compared to annihilation in free open volume.

When He irradiation is performed at the elevated temperature of $600^{\circ} \mathrm{C}$, the broadening and the height of the $\mathrm{S}$ parameter peak becomes much more significant. The maxima 
of these peaks shift to deeper mean positron penetration depth $(220 \mathrm{~nm} / 18 \mathrm{keV})$. These $\mathrm{S}$ parameters more closely reflect the simulated damage profile created by He ion irradiation (Fig. 1). Vacancies generated in tungsten at the irradiation temperature of $600{ }^{\circ} \mathrm{C}$ are mobile and may form large and stable vacancy clusters as indicated by the high $\mathrm{S}$ parameters in Fig. 2(a). ${ }^{1,7,15}$ Figure 2(b) presents the $\mathrm{S}$ parameters of the samples irradiated at RT after post-annealing at $600^{\circ} \mathrm{C}$. For comparison, also $\mathrm{S}$ parameters are plotted for the sample with the highest He fluence of $1 \times 10^{16} \mathrm{~cm}^{-2}$ without post-annealing. After post-annealing, the $\mathrm{S}$ parameters are again significantly enhanced as compared with the corresponding sample without post-anneal. But, the peak of the $\mathrm{S}$ parameters stays in the same low energy (surface) region as for the virgin and RT irradiated samples.

Implanted $\mathrm{He}$ atoms are trapped by the open volume defects in the tungsten, especially by vacancies and vacancy clusters. They form helium-filled vacancy defects of the form $\mathrm{He}_{\mathrm{n}} \mathrm{V}_{\mathrm{m}}{ }^{7,8}$ Such He-vacancy clusters are more stable than the empty vacancy clusters due to $\mathrm{He}$ induced pressurization inside the open volume. Small $\mathrm{He}_{\mathrm{n}} \mathrm{V}_{\mathrm{m}}$ defects $(\mathrm{m}=1)$ are mobile at $600^{\circ} \mathrm{C}$ and tend to coalesce with each other and form the larger $\mathrm{He}_{\mathrm{n}} \mathrm{V}_{\mathrm{m}}$ complexes or even He filled bubbles meanwhile the density of the $\mathrm{He}_{\mathrm{n}} \mathrm{V}_{\mathrm{m}}$ defects decreases. ${ }^{14}$ Therefore, the increase of $\mathrm{S}$ parameters in the HT irradiated samples (Fig. 2(a)) is attributed to the increased mean size of the $\mathrm{He}_{\mathrm{n}} \mathrm{V}_{\mathrm{m}}$ defects. The same effects are also observed in the room temperature implanted sample after the post-annealing with the same temperature $\left(600^{\circ} \mathrm{C}\right)$ and duration $(1 \mathrm{~h})$.

The depth positions of the $\mathrm{S}$ parameter peaks indicate for HT irradiated samples and the formation of stable $\mathrm{He}_{\mathrm{n}} \mathrm{V}_{\mathrm{m}}$ complexes under the ion beam in accordance with the vacancy generation profile (Fig. 1). Whereas in RT irradiated samples, the mobile He atoms are redistributed and preferentially trapped by the ion generated vacancies in the region with low He-to-vacancy ratio $(\mathrm{He} / \mathrm{V}$ in Fig. 1) or by the pre-existing surface defects in the same region. Subsequent annealing results in the growth of these (He-stabilized) vacancy defects and also forms $\mathrm{He}_{\mathrm{n}} \mathrm{V}_{\mathrm{m}}$ complexes closer to the surface.

The S(E) plot implicitly contains the depth distribution of open volume defects. For instance, a numerical solution of the positron diffusion equation with the assumption of a Makhovian implantation profile for the positrons can be applied in order to fit the S(E) to a depth dependent S parameter profile. The software package "VEPFIT,"23 a fast method for such a numerical solution, was used for this purpose. In order to demonstrate more precisely the depth profile of the open volume defects, the $S(E)$ plots for irradiation with $5 \times 10^{15} \mathrm{~cm}^{-2}$ were fitted using the VEPFIT code (Fig. 3(a)). The fit was done assuming box-shaped profiles for layers with different defect structure. In case of the $\mathrm{He}$ implanted samples, 3 boxes could be fitted: a thin surface layer, the irradiation-effected region, and the bulk. Fig. 3(b) shows the VEPFIT results which were achieved for a surface layer extending to $6 \pm 2 \mathrm{~nm}$ (for all fits), and an irradiation-effected region of a width of $236 \pm 16 \mathrm{~nm}$ for the RT as-implanted sample, $243 \pm 7 \mathrm{~nm}$ for the post-annealed

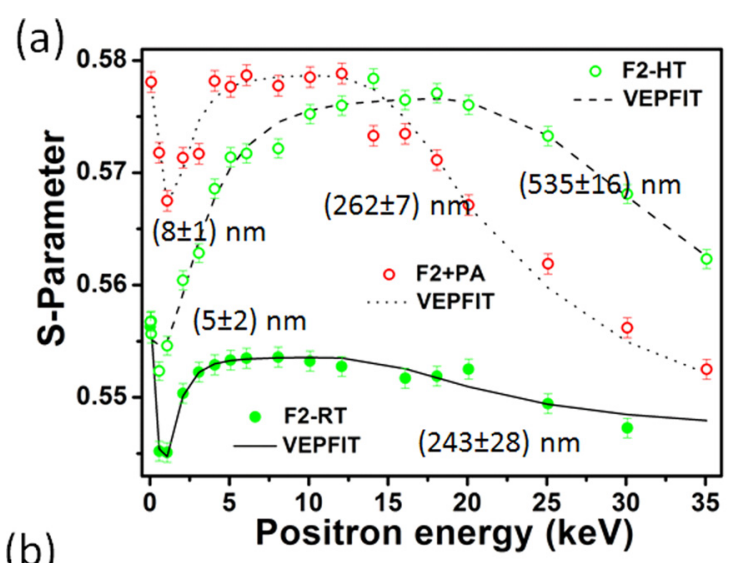

(b)

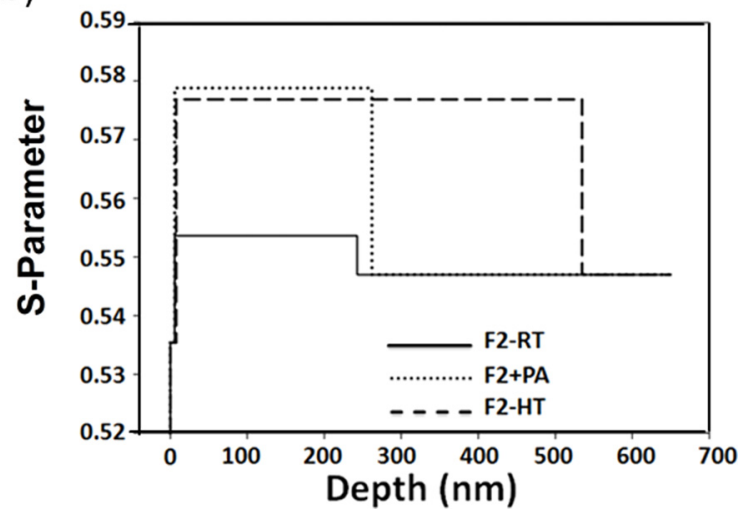

FIG. 3. (a) VEPFIT of the experimental S parameters and (b) depth profiles assuming box-shaped depth profiles consisting of 3 parts, the surface layer with a thickness of $6 \pm 2 \mathrm{~nm}$ (all plots), the irradiation-effected region with a thickness of $236 \pm 7 \mathrm{~nm}(\mathrm{RT}), 243 \pm 28 \mathrm{~nm}(\mathrm{PA}), 538 \pm 16 \mathrm{~nm}(\mathrm{HT})$ and the bulk. Excellent fits to the experimental data are obtained for these depth profiles. The tungsten samples are irradiated by $\mathrm{He}$ with a fluence of $5 \times 10^{15} \mathrm{~cm}^{-2}$ (F2). Irradiation was performed at RT and at $600^{\circ} \mathrm{C}(\mathrm{HT})$. The RT irradiated sample was post-annealed at $600^{\circ} \mathrm{C}(\mathrm{PA})$.

sample, and $536 \pm 28 \mathrm{~nm}$ for the HT implanted sample. By comparison with the TRIM simulation (Fig. 1), it can be seen that the irradiation-effected region extending to about $240 \mathrm{~nm}$ corresponds to the surface-near region with low He-to-vacancy ratio and the extension as far as $536 \mathrm{~nm}$ for the HT irradiation is just in the full depth range to the irradiation damage maximum. Both marks, $240 \mathrm{~nm}$ and $536 \mathrm{~nm}$, are indicated in Fig. 1.

Fig. 4 shows the S-W plot which includes the maximum $S$ values of all $S(E)$ plots. The maximum values are supposed to represent most clearly the dominating type of the open volume defects. All the data points which lie on a straight line represent the same type of open volume defects. ${ }^{2,3,6,7} \mathrm{At}$ least two types of defects can be distinguished either on line L1 or on line L2. The defect type in RT irradiated tungsten (on L1) is clearly different from PA and HT irradiated tungsten (on L2). The higher S parameter of defects on L2 indicates a significantly larger open volume as compared with the defects on L1. In both cases, L1 and L2, the data points shift to the left, to the higher S parameter region, as the $\mathrm{He}$ fluence increases. Basically, all the observed open volume defects in $\mathrm{He}$ irradiated tungsten should be $\mathrm{He}_{\mathrm{n}} \mathrm{V}_{\mathrm{m}}$ defects. The difference between the L1 and L2 defects is supposed to be the very different He-to-vacancy ratio. Assuming that the implanted helium is entirely trapped by the open volume 


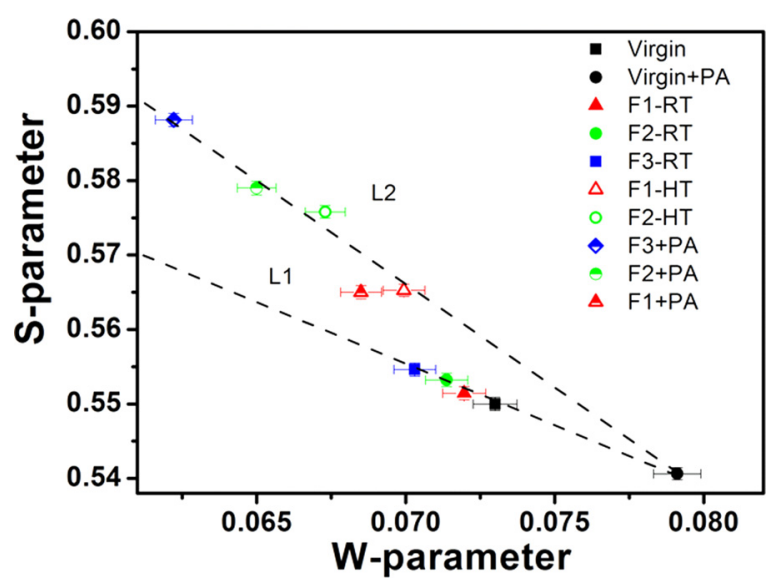

FIG. 4. S-W plot of un-irradiated tungsten (virgin), of un-irradiated and post-annealed tungsten (Virgin + PA), and of He irradiated tungsten at RT with and without post annealing (PA) at $600^{\circ} \mathrm{C}$ as well as of He irradiated tungsten at $600^{\circ} \mathrm{C}(\mathrm{HT})$. The He fluences are $2 \times 10^{15} \mathrm{~cm}^{-2}(\mathrm{~F} 1), 5 \times 10^{15}$ $\mathrm{cm}^{-2}(\mathrm{~F} 2)$, and $1 \times 10^{16} \mathrm{~cm}^{-2}(\mathrm{~F} 3)$. The data are fitted by two straight lines (L1 and L2).

defects without significant out-diffusion, the same amount of $\mathrm{He}$ exists inside the large $\mathrm{He}_{\mathrm{n}} \mathrm{V}_{\mathrm{m}}$ complexes at $\mathrm{L} 2$ as well as inside the smaller vacancy clusters at L1. In consequence, the He concentration inside the L1 defects is much higher. The high He concentration in open volume defects of the RT irradiated samples also results in lower $\mathrm{S}$ parameters. One mono-vacancy in tungsten is known to capture several $\mathrm{He}$ atoms. ${ }^{8,15}$ Of course, the un-irradiated sample also located at L1 does not contain any He. In this case, the open volume surface defects are manufacturing defects (grain boundaries, dislocation loops) as discussed before.

Another process which probably proceeds in RT irradiated tungsten is the He atom self-trapping effect that results in He interstitial clusters. After exceeding the strain threshold, those interstitial clusters emit tungsten matrix atoms and also form $\mathrm{He}_{\mathrm{n}} \mathrm{V}_{\mathrm{m}}$ defects with rather high He content. ${ }^{12,14}$ Although the He concentration in these defects is very high, they still serve in PAS as efficient traps for positrons. ${ }^{24}$

There also exist intrinsic impurities in tungsten such as $\mathrm{C}, \mathrm{Mo}, \mathrm{Cr}$, and $\mathrm{O}$, which more likely form impurity cluster than impurity-vacancy complexes. The mobility of these intrinsic impurities is enhanced at $600{ }^{\circ} \mathrm{C}$ because the dissociation temperature of these complexes is well below $600{ }^{\circ} \mathrm{C}^{12,15}$ They probably are accumulated in the surfacenear region.

\section{Comparison of PAS and NI results}

The impact of the open volume defects on the mechanical properties of tungsten was investigated by comparing the plots of the $\mathrm{S}$ parameters and the hardness values. In Figures 5(a) and 5(b), the PAS and NI results are compared for three samples: virgin tungsten, un-irradiated tungsten after annealing (Virgin + PA), and tungsten after RT irradiation with the highest He fluence of $1 \times 10^{16} \mathrm{~cm}^{-2}$ at RT. The trend or the sequence of $\mathrm{S}$ parameters and hardness values coincide with each other. The highest $\mathrm{S}$ parameters obtained for the $\mathrm{He}$ irradiated sample correlate with the highest hardness values. Annealing of the virgin sample results in lower S parameter

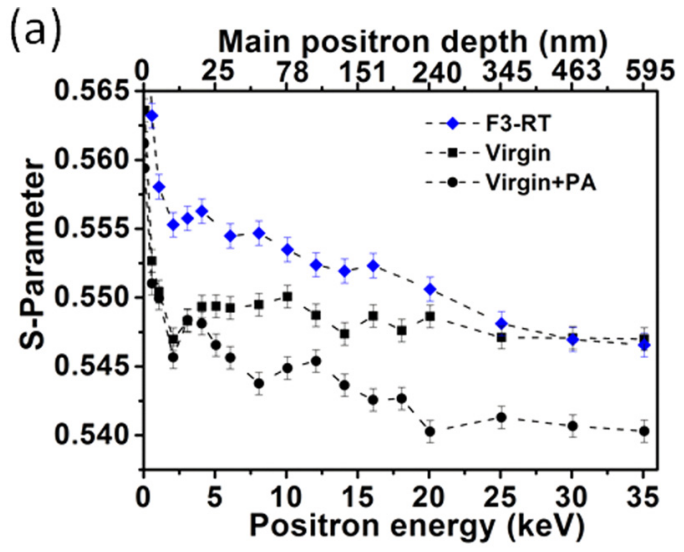

(b)

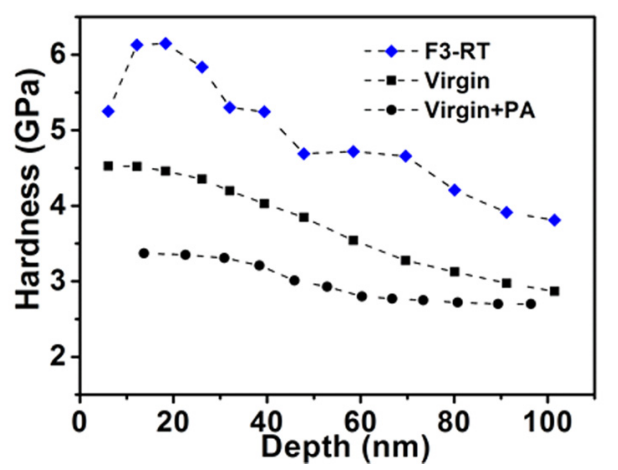

FIG. 5. S parameters as a function of incident positron energy (a) and nanoindentation hardness profiles (b) for virgin tungsten, un-irradiated and postannealed tungsten (Virgin + PA) and He irradiated tungsten at RT with a fluence of $1 \times 10^{16} \mathrm{~cm}^{-2}(\mathrm{~F} 3)$. The annealing of tungsten was performed in vacuum at $600^{\circ} \mathrm{C}$ for $1 \mathrm{~h}$. The hardness data present the average hardness values of 5-8 indentation values. In general, the error of the hardness values is below $5 \%$.

as well as reduced hardness values. S parameters and hardness values are correlated qualitatively. However, their depth profiles and their depth scale deviate from each other. It is important to note that the depth scales of PAS and NI cannot be directly compared. This depth disagreement has been discussed in several previous reports. ${ }^{2,15}$

The differences in the region close to the surface, especially the hardness decline from the surface toward the bulk, can be explained by indentation size effect (ISE). ${ }^{25}$ Due to the lack of the thermal treatment in the virgin sample, the pre-existing manufacturing defects such as impurity clusters, grain boundaries, and dislocation loops located in the surface-near region still have a significant contribution to its hardness. They can serve as pinning sites against the movement of the dislocations. ${ }^{2,15}$ The reduction of density of such defects by the thermal treatment weakens the pining effect and reduces significantly the hardness. On the other hand, if $\mathrm{He}$ atoms are trapped and form $\mathrm{He}_{\mathrm{n}} \mathrm{V}_{\mathrm{m}}$ defects in the same region, hardness and $\mathrm{S}$ parameters are enhanced.

Figures 6(a) and 6(b) show S parameters and hardness values for the thermally treated samples either by HT irradiation or by RT irradiation plus post-annealing. In each case, the thermal treatment of He irradiated tungsten at temperature of $600{ }^{\circ} \mathrm{C}$ results in both, significantly higher S parameters and significantly higher hardness values compared to the virgin $\mathrm{W}$ sample with post annealing (Virgin + PA). 


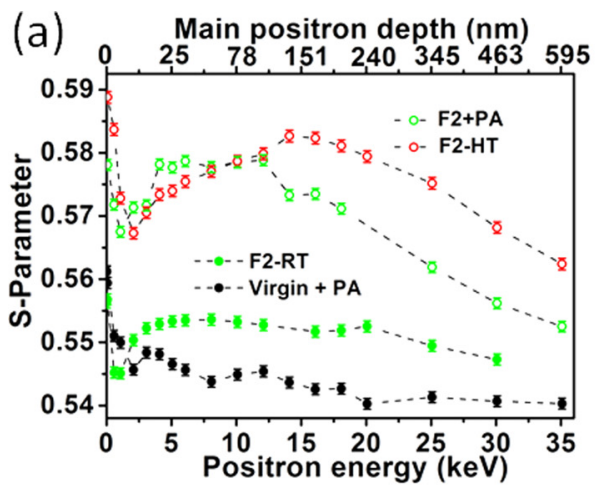

(b)

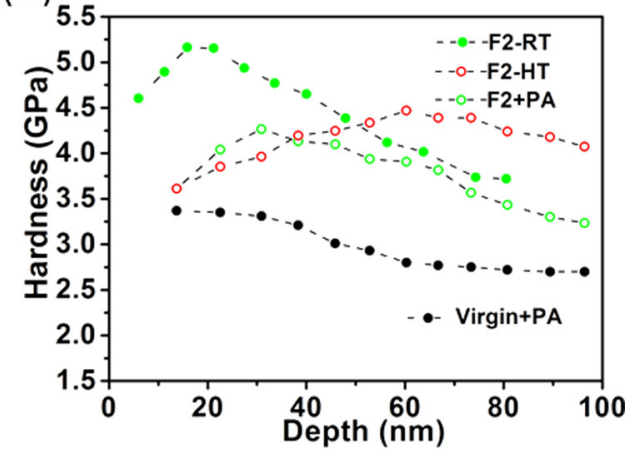

FIG. 6. S parameters as a function of incident positron energy (a) and the corresponding hardness profiles (b) for tungsten. The tungsten samples are virgin, un-irradiated and post-annealed at $600^{\circ} \mathrm{C}$ (Virgin + PA), RT irradiated and subsequently post-annealed at $600^{\circ} \mathrm{C}(\mathrm{PA})$, and irradiated at $600^{\circ} \mathrm{C}$ (HT). All the $\mathrm{He}$ fluences are $5 \times 10^{15} \mathrm{~cm}^{-2}$ (F2). Normalized hardness profiles of the He irradiated samples given by their ratio to the corresponding hardness profiles of the un-irradiated samples (c). Important to notice: The reference sample for the RT irradiated one is the virgin sample (without thermal treatment). The reference sample for the PA and HT samples is the un-irradiated post-annealed sample (Virgin + PA) (with thermal treatment).
Moreover, the different depth distribution of the S parameters observed for HT and PA samples is clearly reflected in the hardness profiles shown in Fig. 6(b). The reason for those different profiles was mentioned above (Sec. II B). In contrast to the HT and PA samples, the sample without any thermal treatment (RT) shows another behavior. The S parameters are lower than those for HT and PA and the absolute hardness values are even higher as compared with the thermally treated samples. This different behavior demonstrates that another type of defects is active and controls the hardness characteristics. Large $\mathrm{He}_{\mathrm{n}} \mathrm{V}_{\mathrm{m}}$ complexes with low He-to-vacancy ratio dominate the behavior of heat treated samples, whereas mainly He-stabilized dislocation loops generate the very high hardness values in RT irradiated samples without post-annealing.

The depth dependent hardness ratio of the irradiated and the non-irradiated sample counts for the ISE, so that irradiation induced hardness increase is better analyzed by relative hardness values. Thus, the intrinsic hardness of the surface layer becomes meaningful as the substrate influence is eliminated. ${ }^{26}$ Figure 6(c) shows normalized hardness values which are the ratio of hardness values of He irradiated tungsten and the hardness of un-irradiated tungsten. The hardness values of virgin tungsten are the reference values for the RT irradiated sample and the hardness values of the unirradiated and annealed sample are the reference for the thermally treated samples, HT and PA. Figure 6(c) clearly underlines the contribution of two different types of defects to the hardening of He irradiated tungsten. At room temperature without any thermal treatment, the irradiation only results in a small hardness ratio despite the measured very high absolute hardness values (Figs. 5(b) and 6(b)). In case of thermally treated tungsten, the hardness ratio is significantly enhanced by the irradiation. This effect is ascribed to the formation of large $\mathrm{He}_{\mathrm{n}} \mathrm{V}_{\mathrm{m}}$ complexes under the thermal treatment whereas without thermal treatment the hardness is controlled by defects showing a low $\mathrm{S}$ parameter such as dislocation loops which are certainly stabilized by the presence of $\mathrm{He}$ atoms. It is also noticed that the relative hardness increase is largest for the irradiated and simultaneously heated tungsten sample. Moreover, the peak of the hardness ratio appears at deeper penetration depth compared to the irradiation induced hardness at room temperature.

\section{SUMMARY}

The distribution and type of open volume defects in $\mathrm{He}$ irradiated tungsten are investigated by positron annihilation spectroscopy and their effect on the hardness of the material is studied by nano-indentation. In detail, the S parameters as a measure for the open volume and the hardness values are compared for the same samples. The results can be summarized as follows:

1) He irradiation of tungsten results in a higher $S$ parameter than in the virgin material. The S parameter is even more enhanced if the sample is heat treated either by irradiation at $600^{\circ} \mathrm{C}$ or by post-annealing at the same temperature after RT irradiation.

2) There are two types of open volume defects: Large $\mathrm{He}_{\mathrm{n}} \mathrm{V}_{\mathrm{m}}$ complexes with low He-to-vacancy ratio formed at $600^{\circ} \mathrm{C}$ and small vacancy clusters with very high $\mathrm{He}$ content created at RT. In case of the high temperature irradiation, the $\mathrm{He}_{\mathrm{n}} \mathrm{V}_{\mathrm{m}}$ complexes are distributed over the whole range of the radiation defect generation. In case of the RT irradiation, small $\mathrm{He}_{n} \mathrm{~V}_{\mathrm{m}}$ defects $(\mathrm{m}=1)$ with high He-to-vacancy ratio form preferentially at the surface-near region where also manufacturing related defects exist. 
3) The hardness characteristics of the heat treated tungsten are clearly related to the measured $\mathrm{S}$ parameter profiles. It means that hardness is controlled by the large $\mathrm{He}_{\mathrm{n}} \mathrm{V}_{\mathrm{m}}$ complexes. Very high hardness values are obtained also for RT irradiated tungsten without thermal treatment. However, this hardness mainly results from pre-existing dislocation loops and small vacancy clusters, both stabilized by a high He atoms and contain a high He content.

4) The relative hardening effect compared to virgin tungsten due to the small $\mathrm{He}_{\mathrm{n}} \mathrm{V}_{\mathrm{m}}$ defects, which are created by RT irradiation, is rather weak. However, the relative hardness values significantly increase as the $\mathrm{He}_{\mathrm{n}} \mathrm{V}_{\mathrm{m}}$ defects grow and become dominant by thermal treatment at $600{ }^{\circ} \mathrm{C}$ during or after He irradiation.

\section{ACKNOWLEDGMENTS}

We gratefully acknowledge support in analyzing the NI measurements by Bodo Wolf, Brandenburgian Technical University Cottbus, Germany.

${ }^{1}$ M. Rieth et al., J. Nucl. Mater. 432, 482 (2013).

${ }^{2}$ R. Kögler, W. Anwand, A. Richter, M. Butterling, X. Ou, A. Wagner, and C. L. Chen, J. Nucl. Mater. 427, 133 (2012).

${ }^{3}$ X. Ou, R. Kögler, H. Zhou, W. Anwand, J. Grenzer, R. Hübner, M. Voelskow, M. Butterling, S. Zhou, and W. Skorupa, Phys. Rev. B 86, 224103 (2012).

${ }^{4}$ L. Thomé and F. Garrido, Vacuum 63, 619 (2001).

${ }^{5}$ Y. Zhang, W. Jiang, C. M. Wang, F. Namavar, P. D. Edmondson, Z. Zhu, F. Gao, J. Lian, and W. J. Weber, Phys. Rev. B 82, 184105 (2010).

${ }^{6}$ A. Debelle, M. F. Barthe, and T. Sauvage, J. Nucl. Mater. 376, 216 (2008).
${ }^{7}$ P. E. Lhuillier, T. Belhabib, P. Desgardin, B. Courtois, T. Sauvage, M. F. Barthe, A. L. Thomann, P. Brault, and Y. Tessier, J. Nucl. Mater. 433, 305 (2013).

${ }^{8}$ C. A. Chen, X. Xiang, Y. Sun, C. L. Zhou, C. X. Ma, and L. Wei, Fusion Eng. Des. 85, 734 (2010).

${ }^{9}$ T. Hino, Y. Yamauchi, and Y. Hirohata, J. Nucl. Mater. 266, 538 (1999).

${ }^{10}$ N. Yoshida, H. Iwakiri, K. Tokunaga, and T. Baba, J. Nucl. Mater. 337, 946 (2005).

${ }^{11}$ S. T. Picraux, Nucl. Instrum. Methods Phys. Res. B 182, 413 (1981).

${ }^{12}$ C. S. Becquart and C. Domain, Phys. Rev. Lett. 97, 196402 (2006).

${ }^{13}$ H. T. Lee, A. A. Haasz, J. W. Davis, R. G. Macaulay-Newcombe, D. G. Whyte, and G. M. Wright, J. Nucl. Mater. 363, 898 (2007).

${ }^{14}$ H. Trinkaus and B. N. Singh, J. Nucl. Mater. 323, 229 (2003).

${ }^{15}$ M. H. Cui, Z. G. Wang, L. L. Pang, T. L. Shen, C. F. Yao, B. S. Li, J. Y. Li, X. Z. Cao, P. Zhang, J. R. Sun, Y. B. Zhu, Y. F. Li, and Y. B. Sheng, Nucl. Instrum. Methods Phys. Res. B 307, 507 (2013).

${ }^{16}$ S. B. Gilliam, S. M. Gidcumb, N. R. Parikh, D. G. Forsythe, B. K. Patnaik, J. D. Hunn, L. L. Snead, and G. P. Lamaze, J. Nucl. Mater. 347, 289 (2005).

${ }^{17}$ W. Anwand, G. Brauer, M. Butterling, H. R. Kissener, and A. Wagner, Defect Diffus. Forum 331, 25 (2012).

${ }^{18}$ A. Richter and R. Smith, Encyclopedia of Nanoscience and Nanotechnology (American Scientific Publishers, 2011), Vol. 17, pp. 375-438.

${ }^{19}$ W. C. Oliver and G. M. Pharr, J. Mater. Res. 7, 1564 (1992).

${ }^{20}$ J. P. Biersack and L. G. Haggmark, Nucl. Instrum. Methods Phys. Res. B 174, 257 (1980).

${ }^{21}$ P. Hosemann, D. Kiener, Y. Wang, and S. A. Maloy, J. Nucl. Mater. 425, 136 (2012).

${ }^{22}$ B. Wolf and A. Richter, New J. Phys. 5, 15.1 (2003).

${ }^{23}$ A. van Veen, H. Schut, J. de Vries, R. A. Hakvoort, and M. R. Ijpma, in Positron Beams for Solids and Surfaces, edited by P. J. Schultz, G. R. Massoumi, and P. J. Simpson (American institute of Physics, New York, 1990), p. 171.

${ }^{24}$ T. Troev, E. Popov, P. Staikov, N. Nankov, and T. Yoshiie, Nucl. Instrum. Methods Phys. Res. B 267, 535 (2009).

${ }^{25}$ G. M. Pharr, E. G. Herbert, and Y. Gao, Annu. Rev. Mater. Res. 40, 271 (2010).

${ }^{26}$ X. Liu, R. Wang, A. Ren, J. Jiang, C. Xu, P. Huang, W. Qian, Y. Wu, and C. Zhang, J. Nucl. Mater. 444, 1 (2014). 